Journal of Teacher Education for Sustainability, vol. 15, no. 1, pp. 48-56, 2013

\title{
A MODEL FOR DEVELOPING META-COGNITIVE TOOLS IN TEACHER APPRENTICESHIPS
}

\author{
Paige Bray \\ University of Hartford, the United States of America \\ and \\ Steven Schatz \\ University of Massachusetts, the United States of America
}

\begin{abstract}
This research investigates a model for developing meta-cognitive tools to be used by pre-service teachers during apprenticeship (student teaching) experience to operationalise the epistemological model of Cook and Brown (2009). Meta-cognitive tools have proven to be effective for increasing performance and retention of undergraduate students. Postulating that the student teaching experience is a new type of learning - learning about practice (knowledge in action), instead of learning curriculum or pedagogy (knowledge possessed) - we suggest that a meta-cognitive tool set may prove similarly useful. Before studying the effectiveness of a tool set, however, a model which enables different programmes to evolve and develop appropriate tools is necessary. This case study research explores a model for the development of a context-specific tool set over 18 months, incorporating user feedback, researcher reflection and multiple-tool development. The model showed promise as a starting point for understanding and operationalising complex interactions with theory and practice.
\end{abstract}

Key words: apprenticeship, teacher education, learning community, case study, metacognitive tools

\section{Introduction}

The culminating experience in teacher education is the student teacher apprenticeship. Traditionally, this is a time when pre-service teachers apply what they have learnt about curriculum and pedagogy to the practice of being a teacher (Britzman, 1991).

However, this model has proven to have some drawbacks. The focus of student teacher and supervisor is largely on transmitting curriculum, not exploring the activity of teaching or the 'risky behaviour' of engaging pupils' learning (Edwards \& Protheroe, 2003). While difficult to teach, the crucial knowledge of how to teach is not learnt by student teachers. The efficacy of the student teacher apprenticeship could be increased by helping pre-service teachers learn the implicit knowledge that in-service teachers use daily, including time management, student interactions and learning how and where to seek information and guidance. 
Studies have shown that meta-cognitive tools, such as study skills and note taking, increase performance and retention of incoming college freshmen (Tuckman \& Kennedy, 2011). Student teachers are faced with a new kind of learning different from college courses. They must learn how to, what to and when to apply skills, knowledge and techniques. Perhaps a different set of meta-cognitive tools could increase efficacy.

Quickly developing and testing the effects of a tool set would be less useful. It would take time and experimentation to develop a working set of tools. However, as there are differences between teacher education programmes, we saw value in developing a systemic model for developing tools, allowing different programmes to share and discuss the results and impact of tools. The field is best served by studying a model for developing tools. Then, with the model in place and tools developed, research on efficacy can proceed in the future. The research described in this paper is a case study of the evolution of a model for developing a set of meta-cognitive tools.

Our work was greatly influenced by the epistemological model of Cook and Brown (1999) who postulate two types of knowledge - knowledge that is possessed (I know how to add $2+2$ ) and knowledge that can only be accessed through action (I know when to shift the gears in a car, what my child's cry means, but only when I am in the activity). Teacher preparation course work focuses on knowledge of possession. However, much of the practice of teaching relies on knowledge that is based in action. An experienced teacher knows when to push, repeat or encourage based on the moment and the situation. The knowledge of how to be a teacher is inextricably tied to the activity of teaching.

The student teacher learns from watching and, in part, from Legitimate Peripheral Participation (Lave \& Wenger, 1991). However, instead of a focus on reflection on practice as an end in itself, we sought to use reflection on practice to explore knowledge of practice as well as methods and sources for seeking new information knowledge. The aim is not for teachers to ask a question: How did I do? but Where can I find more to improve my knowledge of my practice? We hoped this would facilitate consideration and transfer of learning between students during the seminar and would carry forward as a learned behaviour into the students' professional lives, encouraging the formation of communities of practice to support continued learning.

This research addresses the following questions:

1. How can the epistemological model of Cook and Brown be operationalised in a meta-cognitive tool set for use by student teachers?

2. Can we develop a model for developing tools that allows the tools to evolve over time in a theoretically sound and systemic way which can be applied and studied by other programmes of teacher education? What are the features of this model of development?

3. What tool set results from questions one and two above?

\section{Methodology}

This is case study research (Yin, 2002) based in an objectivist epistemology and a postpositivist methodology (Crotty, 1998; Phillips \& Burbules, 2000) in a naturalistic setting. The unit of study is both the researchers developing a set of meta-cognitive tools and the model for development of those tools. We study and present here the method used for evaluating the tools and the evolution of the tools. We expect that 
other researchers will find need for different tools. We expect the tool set will change over time, as needs change, sometimes because of the implementation of the tools. We report on the evolution of the tools only as they inform the model for development of such tools.

As case study research, we do not claim that the tool set developed can be generalised to the population of student teachers or to other programmes of teacher education. We cannot claim the model for development can be generalised. Every programme will adjust the model and tool set to meet their specific needs. However, this case provides a starting point and process for beginning to understanding the complex interaction between pedagogy, epistemological stances and research on student teaching experiences.

The research was carried out by two researchers. One, a participant/researcher, taught the seminar class required during the student teaching experience. The other, not associated with the university, took the role of outside 'foil' to reflect upon the practice of using the tool set. In addition, as the outside researcher had no influence on student evaluation, it was possible to review student feedback gathered through online questionnaires during the semester. The outside researcher could view answers and check to make sure responses were complete, while ensuring anonymity for students. The participant/researcher only saw data without names of students and then only after the semester was over.

\section{A model for development}

The model for development of meta-cognitive tools studied during this research was as follows:

- Initial tool development. Reading and discussions between the researchers focused on methods to operationalise the epistemological model of Cook and Brown, with guidance from the literature of apprenticeship, teacher practice and success of meta-cognitive tools.

- Feedback. We believed our first attempts to develop tools would need alteration, so we developed feedback mechanisms that could provide input during implementation and could be analysed at the end of the semester. These included online surveys from students, observations by the participant researcher and reflections by both researchers. The participant researcher's entries specifically noted instances that indicated student teachers' information seeking from new venues and transferring the lessons learned from specific incidents to overall practice. The outside researcher focused on decisions made and changes to tools, feedback mechanisms and instructions for assignments.

- Researcher reflection and discussion during semester. Both researchers engaged in regular discussions throughout the semester, specifically discussing methods to introduce and explain tools and assignments, how much and the nature of modelling and guidance to be given by the participating research during the seminar as well as the significance of incidents and activities of student teachers.

- Debriefing. At the end of each semester, the researchers held an extensive debriefing session, discussing what was learned, what worked well, where problems arose, incidents which offered evidence of impact and how the research was informing the underlying epistemological framework. 
- Set future changes. At the end of every semester, the researchers made adjustments to the tool set, instructions for students and research methods. Based on Alexander's work (Alexander, 1979), we believe that the development of any model is an ongoing process of 'repair'. Based on how the tools were used, we developed changes for the next semester.

\section{Findings}

The programme studied was an initial teacher certification (undergraduate and graduate programmes) in Early Childhood Education (ECE) at a small, private Eastern United States university. The department graduates about 100 students in all programmes. The ECE programme graduates about 25 teacher candidates per year.

Teacher candidates must complete two semester-long student teaching experiences before graduation. During the first semester, students are placed in a pre-kindergarten or kindergarten classroom for a half-day, 20-hour per week experience. They are overseen by a cooperating teacher in the classroom and a university supervisor who observes them five times during the semester. In addition, they must attend a weekly seminar on teaching methods. The tool set was explained and used in this seminar.

During the second semester, the student teaching experience is full day, 40-hour per week in either infant/toddler or forms 1-3, depending on the student's concentration. During the second semester, the seminar alternates between in-person and online meetings.

This research into the model for developing meta-cognitive tools extended over three semesters. Below we explore how the model was used in each semester.

\section{Semester one}

Tool development. Initially, we used faculty awareness of Cook and Brown's epistemology, coupled with explicit statements to teacher candidates about the nature of the student teaching experience. The following statements were used repeatedly: This is not a test of your skills, but a new kind of learning-learning in action. Learning about practice. This is different from taking a class. You will continue to learn this way throughout your teaching career. You may find it difficult, even if you have found classes to be easy. There is no clear right way to do most things. In this seminar, you begin a continual improvement of your practice as a teacher.

The advantage of using awareness as a tool is that it is an inexpensive and easy intervention, not requiring systemic or curricular changes. Faculty leading the seminar need to gain as well as communicate a new understanding.

Feedback. To gather information from student teacher candidates, we developed and implemented an online survey, using 18 questions - most open-ended, asking students to discuss and reflect upon an experience during teaching that provided a challenge to their practice. The survey was administered at the end of the semester. Researchers' observational journals were maintained.

Researchers' reflection and discussion during semester. The researchers gave consideration to having the outside researcher present Cook and Brown's model explicitly to the seminar. We decided a theoretical discussion would confuse, not serve the students 
in the midst of the student teaching experience. We opted for learning through guided action and practice.

The practicing researcher realised that she was guiding students to answer questions about practice, but the students were not initially learning what questions they could ask of themselves or each other, that could be applied in future situations. For instance, she guided students to identify authentic issues of practice, distinguished from complaints. Then, using clarifying and guiding questions, she elicited further description and detail, demonstrating that responses, feedback and answers were all useful in gaining understanding and formulating strategies for this and other issues. Students, with such guidance, began to understand that there is most often not one solution, but progress towards resolution of a specific issue. In discussions, the participant researcher stressed her observing the importance of student teachers' experiencing these explicit, collaborative, interactive opportunities in order to 'own' their learning, to act on it and learn from and with others.

In exchanges about this issue, we saw the need to create an explicit tool with intentional activities, requiring use and fostering understanding, which would encourage developing practice and skills that could be transferred to other situations and could be used throughout the teaching career.

Debriefing. Upon review of the semester, some key decisions were reached. Some students were confused by the directions for certain questions in the survey. It was clear a survey used as a pre- and post-assessment would yield more informative data.

We decided to create a meta-cognitive tool, an assignment with an explicit discussion protocol. Each student would identify an issue that they faced during their student teaching practice and would present it, guided by the following protocol:

1. Describe the problem.

2. What did you do?

3. Whom did you ask for help or feedback?

4. Upon reflection - whom else would you like to have talked with? What other sources of information could/would you like to have used (resources, people, materials, professional organisations)?

5. Upon reflection - would you have done something different? What? Why?

6. Questions/What do I need from the seminar group?

Each student completed this assignment once in the semester, one student presenting each week. Fellow students would offer feedback and make connection to their own practice.

The participant researcher explicitly modelled and directed students to understand that this was not just one more assignment to be completed and forgotten, but a metacognitive tool that could improve practice and inform their actions in the future.

The second tool discussed was an online discussion board. We wanted to extend the class discussion beyond the boundaries of the seminar meeting time, into the realtime student teaching experience. We decided to establish an online discussion board and resource depository. The hope was the assignments would be posted between meeting times and other members of the seminar could read, reflect and write within that forum. We also hoped that after graduation, this board would become an ongoing forum for discussing issues and sharing resources among pre-service and in-service teachers.

Set future changes. These changes to the tool set and instructions are the implementation of decisions reached during the debriefing stage. The previous step looked 
backward as the researchers revisited what occurred. This step looks forward to implementation during the coming semester.

The following changes were implemented before the beginning of the second semester:

- implementing online discussion board, not using the university's learning management system, to encourage the creation of a repository of discussion and resources during and after student teaching;

- clarifying instructions for survey instrument (specific incidents queried were specific to teaching situations);

- implementing pre- and post-administration of online survey.

\section{Semester two}

Tool development. With tool changes described above, the major change in practice for the participant researcher was implementation of the explicit discussion protocol. During each seminar session, one student presented an authentic issue for discussion that arose during the student teaching experience using the above protocol to prepare themselves in advance. The participant researcher established norms for the depth of presentation, level of feedback and richness of the discussion. The presenter was graded, a web-based discussion board was created, and the revised survey was administered at the beginning and end of the semester.

Feedback. Pre- and post-surveys administered, researchers' observational journal continued.

Researchers' reflection and discussion during the semester. The participant researcher was concerned that students would be simplistic in their answers and cursory in their responses during discussion. However, the students were compelled by the authentic issues. The added scaffolding of modelling presentation and reinforcing active listening by the participant researcher was of value. In addition, guidance was provided to selfmonitoring the amount of talk time and hearing from all seminar participants.

Extensive reflection and discussion by students supported the decision to provide active modelling by the participant researcher, then fading out her primary role as the modelling and feedback were shared by all seminar participants.

Debriefing. The discussion board did not work as an online venue. It was another forum and online location, so became more of an assignment to complete. We decided to use the discussion forum integrated with the learning management system that the students were already using, opting for greater use during the seminar and forgoing the possibility of in-service teacher input after graduation.

We decided to add a new tool to integrate with an existing key assessment. The seminar already required a teacher inquiry project where students formally formulated a question, utilised multiple data sources, analysed, reflected upon and presented their inquiry during the final seminar session. A formal rubric assessment was added to the tool set in order to assess the semester-long, iterative process. Students were required to make explicit connections between what was learned during their presentation, discussion and the inquiry project. By doing this, we hoped to enrich both the discussion and the inquiry project. 


\section{Future changes:}

- change instructions of inquiry key assessment to add rubric to the tool set;

- use university-linked system for discussion board;

- formalise explicit instructions as developed during semester discussion.

\section{Semester three}

Tool development.

- implement new instructions for inquiry assessment and rubric for evaluation;

- implement a new discussion board;

- implement instructions for discussions.

Feedback. Pre- and post-surveys and researchers' observational journal continued. Key assessment data of teachers' inquiry were made available at the end of the semester, as well as the incorporated discussion board content was made available for review.

Researchers' reflection and discussion during the semester. Participant researchers' entries in the observational journal note the students' taking greater responsibility for guiding their conversation at the beginning of in their full day student teaching experience and by mid-semester stewarding their own conversations with minimal participant researcher facilitation.

Debriefing. We confirmed our confidence that the tool set - the explicit discussion protocol, the discussion board and the teacher's inquiry assignment - was now stable. We recognised the feedback - student pre- and post-survey, researchers observational journal and documentation created via the discussion board - was also functioning well, although we desired more perspectives.

We recognised a need to provide guidance for students to initiate discussions about practice with their supervising teachers. As these professionals are used to discussing curriculum transmission and are in a supervisory role, it is often not easy for a student to steer the direction of a conversation into less certain waters. We felt that an assignment requiring students to ask their supervising teacher about a specific practice would help. During the coming semester, we will develop a new tool, perhaps as follow-up to the students' presentation that requires the student to broach this subject.

\section{Future changes:}

- begin to develop a tool for students asking teachers about practice;

- expansion of feedback to supervising teachers and cooperating teachers;

- outreach to graduated, in-service teachers to gather anecdotal evidence of changes in practice.

\section{Conclusion}

Based on this research, we have found the above model for developing meta-cognitive tools shows promise. At this point, we believe the tool set, now in its third iteration, is rich and relatively stable. The model has provided a useful framework for developing tools and researchers' reflecting upon their effectiveness. Student teachers engaged in the seminar have been observed demonstrating an increased awareness of their practice and have employed what they have learned when participating in discussions with other seminar members. 
One of the strengths of the model for tool development studied is the in-process reflection of tools followed by a summative debrief. This two-pronged approach enabled us to determine what needs the students exposed while considering the overarching theoretical application. In attending to both the practical and the theoretical, we affirmed that some tools worked, some were not effective.

We continued to see the power in our decision not to talk about theoretical frameworks within the seminar, but rather make explicit the theoretical grounding/framework through assignments, activities and experiences - operationalising the theory.

We have seen the importance of not merely providing directions for assignments, but, instead, using clear, explicit modelling and directed feedback to support yet intentional reflection and discussion, which moves the focus from completing to enacting the assignment. Within this case, modelling led to rich discussions of the importance of effective teaching, thinking about learning and, by listening to each other, seeing ways to apply knowledge in new contexts.

While not explicitly addressed in either model or tool set, the participant researcher worked to empower the students in the seminar to form a learning community. Through instruction, facilitation and feedback, the researcher set expectations for the seminar to have a less hierarchical structure than is typical. Group norms included: increased accountability, active listening, respective feedback and responsive engagement by each seminar student. An important result of these norms was that students took responsibility for their own and their colleagues' learning. They assumed accountability to each other.

Finally, an important lesson learned was taking time to implement and consider the effect of the tools. In some cases, the tools were not weak, but the students needed time to apply them over two semesters in two distinct school contexts and seminars in order to understand their usefulness. It was in the second semester, when the students where familiar with the tool, that they were able to reap more complex and complete rewards. Related to this finding is the importance of multiple uses of each tool with guidance. We believe the effectiveness of the tools are enhanced by the duration of engagement and facilitated by the length of time provided to revisit overtime rather than limit engagement with a short-term assignment. The goal is a change in practice over a career, not the completion of a single assignment.

Based on this case, this model for the development of a meta-cognitive tool set shows promise for further study. Two areas to investigate in future research include: efficacy of the tool set on student teachers' practice as measured by feedback from classroom cooperating teacher, university supervisor and the student, effect on the P-12 student learning and further evolution of the tool set and an application of the model in other programmes by other programme-based researchers.

\section{References:}

Alexander, C. (1979). The timeless way of building. New York: Oxford University Press.

Britzman, D. (1991). Practice makes practice: A critical study of learning to teach. Albany: State University Press.

Cook, S. D. N., \& Brown, J. S. (1999). Bridging epistemologies: The generative dance between organizational knowledge and organizational knowing. Organization Science, 10(4), 381-400. DOI: 10.1287/orsc.10.4.381. 
Crotty, M. (1998). The Foundations of social research: Meaning and perspective in the research process. London: Sage Publications.

Edwards, A., \& Protheroe, L. (2003). Learning to see in classrooms: What are student teachers learning about teaching and learning while learning to teach in schools? British Educational Research Journal, 29(2), 227-242. DOI: 10.1080/01411920 32000060957.

Lave, J., \& Wenger, E. (1991). Situated learning: Legitimate peripheral participation. Cambridge: Cambridge University Press.

Phillips, C. C., \& Burbules, N. C. (2000). Postpositivism and education research. Lanham: Rowman \& Lettlefield.

Tuckman, B. W., \& Kennedy, G. J. (2011). Teaching learning strategies to increase success of first-term college students. The Journal of Experimental Education, 79(4), 478-504. DOI: 10.1080/00220973.2010.512318.

Yin, R. K. (2002). Case study research: Design and methods (3rd edition). Newbury Park: Sage Publications.

\section{Correspondence:}

Paige M. Bray, Assistant Professor of Early Childhood, Early Childhood, University of Hartford, Hillyer 232, 200 Bloomfield Avenue, West Hartford, CT 06117. Email: Bray@hartford.edu or schatz@powerstart.com 